Research Paper

\title{
Changes in Bone Density after Cancer Treatment in Patients with Cervical and Endometrial Cancer
}

\author{
Young Lim Oh${ }^{1}$, Man Soo Yoon², Dong Soo Suh², Ari Kim³, Min Joung Kim4, Ji Young Lee ${ }^{5}$, Yong Jung \\ Song ${ }^{6}$, Yong Il Ji ${ }^{7}$, Ki Hyung Kim ${ }^{2 \star}$, and Sungwook Chun ${ }^{7 * \llbracket}$ \\ 1. Department of Obstetrics and Gynecology, College of Medicine, Kosin University, Busan, Republic of Korea; \\ 2. Department of Obstetrics and Gynecology, Pusan National University School of Medicine, Busan, Republic of Korea; \\ 3. Department of Obstetrics and Gynecology, Institute of Wonkwang Medical Science, College of Medicine, Wonkwang University, Iksan, Korea; \\ 4. Department of Obstetrics and Gynecology, Catholic University School of Medicine, Seoul, Republic of Korea; \\ 5. Department of Obstetrics and Gynecology, Konkuk University School of Medicine, Seoul, Republic of Korea; \\ 6. Department of Obstetrics and Gynecology, Pusan National University School of Medicine, Yangsan, Republic of Korea; \\ 7. Department of Obstetrics and Gynecology, College of Medicine, Inje University, Busan, Republic of Korea. \\ * Ki Hyung Kim and Sungwook Chun equally contributed to this article for correspondence.
}

$\triangle$ Corresponding authors: Ki Hyung Kim, MD, PhD, Department of Obstetrics and Gynecology, Pusan National University School of Medicine, and Biomedical Research Institute and Pusan Cancer Center, Pusan National University Hospital, 179 Gudeok-ro, Seo-gu, Busan 602-739, Republic of Korea. Tel: +82-51-240-7287; Fax: +82-51-248-2384. E-mail: ghkim@pusan.ac.kr or Sungwook Chun, MD, PhD, Department of Obstetrics and Gynecology, College of Medicine, Inje University Haeundae Paik Hospital, 875, Haeun-daero, Haeundae-gu, Busan 612-030, Republic of Korea. Tel: +82-51-797-2020; Fax: +82-51-797-2030; E-mail: wooki1974@empal.com.

(c) Ivyspring International Publisher. This is an open-access article distributed under the terms of the Creative Commons License (http://creativecommons.org/ licenses/by-nc-nd/3.0/). Reproduction is permitted for personal, noncommercial use, provided that the article is in whole, unmodified, and properly cited.

Received: 2014.09.30; Accepted: 2014.11.14; Published: 2015.01.01

\begin{abstract}
OBJECTIVE: This study aimed to evaluate the impact of cancer treatment on bone mineral density (BMD) in the lumbar spine (LS) and femur in the postmenopausal women with cervical or endometrial cancer without bone metastasis compared to normal control postmenopausal women.

METHODS: We retrospectively evaluated the BMD data in the LS, femur neck (FN) and trochanter (FT) by dual-energy X-ray absorptiometry and laboratory data of bone turnover markers at baseline and after one year in 130 patients with cervical cancer, 68 patients with endometrial cancer, and 225 healthy controls.

RESULTS: There were no significant differences in the T-scores of basal BMD in LS and femur between patients with endometrial cancer and controls, and only T-score of basal BMD at the fourth lumbar vertebra (L4) was significantly lower in patients with cervical cancer compared to controls. One year later, T-scores of BMD at all LS sites and FN in patients with cervical cancer and T-scores of BMD at L3, L4, FN, and FT in those with endometrial cancer after cancer treatment were significantly lower compared to controls. Lower proportions of normal BMD at all skeletal sites except L2 in patients with endometrial cancer and those at L1, L4, and FN in patients with cervical cancer were observed compared to controls after cancer treatment.
\end{abstract}

CONCLUSIONS: Our results suggest that cancer treatment increase bone loss in postmenopausal women with cervical and endometrial cancer.

Key words: Bone mineral density; Cervical cancer; Endometrial cancer; Osteoporosis.

\section{Introduction}

Osteoporosis is defined as a skeletal disorder characterized by compromised bone strength which is an integration of bone density and bone quality, pre- disposing to an increased risk of fractures associated with chronic pain, disability, and mortality [1]. Estrogen has a key role of maintaining a balance between 
osteoblastic and osteoclastic activity, and bone remodeling increases when estrogen levels decline [2]. Hormone levels are the main determinants of bone density, but other factors including the presence of other medical conditions also increase the risk of osteoporosis [3-5].

Many cancer patients were confronted to cancer-related bone loss and development of osteoporosis in their lives, and these results are mainly caused by; 1) osteolytic factors by cancer cell lines themselves [6]; and 2) bone loss induced by anti-cancer treatment which is called cancer treatment-induced bone loss (CTIBL) [7].

A variety of neoplasms without bone metastasis are also known to be related to osteoporosis by producing circulating bone resorption stimulatory factors leading to bone destruction and hypercalcemia [6, 8-12]. In patients with gynecologic cancers, reduced spinal bone mineral density (BMD) has been reported in patients with cervical cancer [13, 14], but no significant differences were observed in lumbar or femoral BMD between patients with endometrial cancer and controls [15].

CTIBL may cause bone fragility and an increased susceptibility to fractures, and bone loss occurs more rapidly and tends to be more severe in patients with CTIBL than in those with normal age-related bone loss; therefore, prevention, early diagnosis, and treatment of CTIBL are essential to decrease the risk of fracture [7, 16, 17]. As estrogens act through direct and indirect mechanisms to restrain bone resorption, all oncology therapies that induce hypogonadism cause osteoporosis in a large percentage of patients [17]. CTIBL is most common in patients with breast or prostate cancer who receive chemotherapy, hormone therapy, or surgical castration, as these can cause hypogonadism and induce bone loss $[7,17]$. There have been a few studies reported about the association between gynecologic cancer treatment and bone loss $[18,19]$.

The aim of the present study was to evaluate the impact of cancer treatment on BMD in the lumbar spine (LS) and femur in the postmenopausal women with cervical or endometrial cancer without bone metastasis compared to normal postmenopausal women.

\section{Materials and Methods}

\section{Subjects}

Postmenopausal women aged 45-57 years who first visited Kosin University Gospel Hospital and were diagnosed with cervical or endometrial cancer without bone metastasis between January 2005 and December 2010 were included in the study. This study was approved by the Institutional Review Board of Kosin University Gospel Hospital, and all subjects provided their informed consent. All those with cervical or endometrial cancer who had not reached menopause or who received menopausal hormone therapy were excluded in this study. Menopause was defined as no spontaneous menstruation for more than 12 months. Study subjects who had used drugs known to alter bone or calcium metabolism were also excluded in this study.

Cervical cancer was diagnosed by Papanicolaou smear and colposcopically directed biopsy. All patients who were diagnosed with cervical cancer underwent Type II or III hysterectomy, bilateral salpingo-oophorectomy (BSO), and pelvic lymphadenectomy, and dissections of suspicious para-aortic lymph nodes by intraoperative examination were also made. Most of patients received adjuvant concurrent chemoradiation therapy (CCRT) using platinum-based chemotherapy after surgery.

Endometrial cancer was initially diagnosed by dilatation and curettage of the uterus. Technetium-99m-labeled diphosphonate bone scans or 18F-fluorodeoxyglucose positron emission tomography/computed tomography (PET/CT) were performed on all cancer patients for confirmation of bone metastasis. All patients who were diagnosed with endometrial cancer underwent Type I or II hysterectomy with bilateral salpingo-oophorectomy, washing cytology, and pelvic and/or para-aortic lymph node dissection, followed by internal or external radiation therapy to the pelvis. Finally, 130 patients with cervical cancer and 68 patients with endometrial cancer were enrolled in this study. 225 postmenopausal women aged 48-59 years who visited the University Hospital annually as part of a group check-up for work and lacked specific health problems served as normal controls. All control women underwent a careful physical examination and a thorough review of medical history at first visit, and the subjects who had history of current treatment with drugs known to alter bone or calcium metabolism were excluded.

All of study participants received dual-energy X-ray absorptiometry (DEXA) on LS and femur and blood test for bone turnover markers at the time of diagnosis before any cancer treatment, and all of them received follow-up DEXA and laboratory tests of bone turnover markers 1 year later, which is the time after completion of cancer treatment. BMD data of the LS and femur, and laboratory data of bone turnover markers at initial check-up and those in one year were collected for all participants.

\section{Measurements of BMD}

BMD in grams per square centimeter and 
T-scores in the LS (L1-L4) and the left femur neck (FN) and femur trochanter (FT) were determined using a dual-energy X-ray absorptiometer (Lunar Radiation Corp, Madison, WI, USA). BMD values were categorized into three groups according to the criteria of the World Health Organization [20] as normal, osteopenic, or osteoporotic relative to the mean and standard error (SE) of young adult Korean women. The in vivo coefficients of variations were all below $2.0 \%$.

\section{Measurement of serum calcium, phosphorus, osteocalcin, and total alkaline phosphatase, and urine deoxypyridinoline}

Blood samples were collected from an antecubital vein in all the subjects in tubes without anticoagulants, in accordance with the Declaration of Helsinki guidelines, and sera were obtained by centrifugation for determination of bone turnover markers. Urine samples from all participants were also collected. Calcium (Ca) and phosphorus $(\mathrm{P})$ were measured by atomic extinction photometry. Serum osteocalcin (OST) was measured using a NovoCalcin kit (Metra Biosystems Inc., Mountain View, CA, USA). Total alkaline phosphatase was measured using the Kind and King method. Urine deoxypyridinoline (DPL) was measured using a Pyrilink-D kit (Metra Biosystems Inc., Mountain View, CA, USA). Intraand interassay coefficients of all variation were all below $8.0 \%$.

\section{Statistical analyses}

The statistical software package SPSS 18.0 (SPSS Inc., Chicago, IL, USA) was used for all data analyses. All data were expressed as mean \pm standard deviation (SD). For comparisons of demographic and anthropometric characteristics, serum and urine biochemical markers, and T-scores of basal BMD between patients with gynecologic cancers and controls, one-way analysis of variance (ANOVA) and unpaired $t$-test were performed. For comparison of these parameters in cancer patients categorized according to cancer stage, unpaired $t$-test and Kruskal-Wallis test were used. The frequencies of osteoporosis, osteopenia, and normal BMD according to basal bone mass were compared between patients with gynecologic cancer and controls using the chi-square test. $P$ values $<0.05$ were considered significant for all analyses.

\section{Results}

In 130 patients with cervical cancer, the distribution of the International Federation of Gynecology and Obstetrics (FIGO) stage was IB, 73 (56.2\%) and IIA, $57(43.8 \%)$. Of these patients, 103 had squamous cell carcinoma, 18 had adenocarcinoma, 6 had adenosquamous carcinoma, and 3 had other types of cancer. In 68 patients with endometrial cancer, the distribution of surgical FIGO stage was IA, 54 (79.4\%); IB, 7 (10.3\%); IIA, 6 (8.8\%); and IIB, 1 (1.5\%). Of these patients, 53 had endometrioid adenocarcinoma, 10 had squamous cell carcinoma, and 5 had papillary serous adenocarcinoma.

Table 1 shows demographic and anthropometric characteristics in three groups. Age, body mass index (BMI), parity, and time since menopause did not differ significantly between the three groups. Only the T-score of basal BMD at L4 was significantly lower in patients with cervical cancer compared to controls (Table 2), and significant difference in the proportion of osteoporosis according to basal BMD was also observed only at L4 between patients with cervical cancer and normal controls (Table 3). At 1 year, with completion of cancer treatment, T-scores of BMD at all skeletal sites examined except FT were significantly lower in patients with cervical cancer compared to controls, and the proportions of normal BMD at L1, L4, and FN in patients with cervical were significantly lower than those of controls. Table 4 and Table 5 show no significant differences in the T-scores of basal BMD and the proportion of osteoporosis in the lumbar spine and femur between patients with endometrial cancer and normal controls. However, after cancer treatment, T-scores of BMD at L3, L4, FN, and FT of patients with endometrial cancer were significantly lower than those of controls, and the proportions of normal BMD at all skeletal sites except L2 in patients with endometrial cancer were significantly lower compared to controls.

Among the biochemical markers measured, serum calcium and urine DPL levels were significantly higher in patients with cervical cancer compared to controls at baseline (Table 2). After cancer treatment, serum OSC and urine DPL levels are significantly higher than those of controls. No statistically significant differences in any of serum markers were detected between patients with endometrial cancer and controls at baseline, however, serum ALP levels in patients with endometrial cancer were different from those in controls after 1 year (Table 4 ).

Table 1. Comparisons of demographic and anthropometric characteristics between patients with cervical cancer, those with endometrial cancer, and controls.

\begin{tabular}{|c|c|c|c|c|}
\hline & Control & Cervical Cancer & $\begin{array}{l}\text { Endometrial } \\
\text { cancer }\end{array}$ & \multirow[t]{2}{*}{$\begin{array}{l}\mathrm{P} \\
\text { value }\end{array}$} \\
\hline & $\mathrm{N}=225$ & $\mathrm{~N}=130$ & $\mathrm{~N}=68$ & \\
\hline Age (years) & $51.81 \pm 2.46$ & $51.83 \pm 3.29$ & $51.75 \pm 3.33$ & 0.982 \\
\hline Parity & $2.40 \pm 1.54$ & $2.08 \pm 1.48$ & $2.07 \pm 1.49$ & 0.092 \\
\hline $\begin{array}{l}\text { Years since meno- } \\
\text { pause (years) }\end{array}$ & $1.76 \pm 1.18$ & $2.12 \pm 1.93$ & $2.07 \pm 1.92$ & 0.077 \\
\hline $\begin{array}{l}\text { Body mass index } \\
\left(\mathrm{kg} / \mathrm{m}^{2}\right)\end{array}$ & $24.40 \pm 2.35$ & $24.21 \pm 3.56$ & $24.20 \pm 3.41$ & 0.787 \\
\hline
\end{tabular}

Values are mean \pm standard deviation. $P$ values by ANOVA 
No significant differences in clinical, laboratory, or BMD data were observed among patients with cervical cancer divided according to cancer stage before and after cancer treatment, and no differences in these parameters were found among patients with endometrial cancer divided according to stage before and after cancer treatment (data not shown).

\section{Discussion}

Many treatments used in gynecologic oncology have the potential to damage bone, and osteoporosis is therefore one of the long-term complications of successful tumor treatment. Best results for osteopo- rosis treatment can be achieved in the early stages of osteoporosis, and the key to reducing the fracture rate in patients with therapy-induced osteoporosis lies in the early recognition of such complications $[17,21$, 22]. There are no evidence-based guidelines for diagnostic evaluation of patients at risk for CTIBL, but BMD by DEXA is considered the best predictor of fracture risk in general [21]. In the present study, T-scores of BMD of the LS and femur in the patients with cervical and endometrial cancer were significantly lower compared to controls after cancer treatment.

Table 2. Comparisons of serum and urine biochemical markers, and T-scores of bone mineral density in the lumbar spine, femur neck, and femur trochanter at baseline and after one year between patients with cervical cancer and controls.

\begin{tabular}{|c|c|c|c|}
\hline & Control & Cervical Cancer & $P$ value \\
\hline & $\mathrm{N}=225$ & $\mathrm{~N}=\mathbf{1 3 0}$ & \\
\hline \multicolumn{4}{|c|}{ At baseline } \\
\hline \multicolumn{4}{|l|}{ Biochemical markers } \\
\hline $\mathrm{Ca}(\mathrm{mg} / \mathrm{dL})$ & $9.446 \pm 0.219$ & $9.493 \pm 0.208$ & 0.049 \\
\hline$P(\mathrm{mg} / \mathrm{dL})$ & $3.745 \pm 0.237$ & $3.719 \pm 0.182$ & 0.254 \\
\hline OSC (ng/ml) & $17.342 \pm 2.303$ & $16.903 \pm 1.744$ & 0.054 \\
\hline $\operatorname{ALP}(\mathrm{IU} / \mathrm{L})$ & $122.524 \pm 4.713$ & $122.792 \pm 3.875$ & 0.563 \\
\hline DPL (pmol/umol creatinine) & $8.340 \pm 0.817$ & $8.542 \pm 0.740$ & 0.018 \\
\hline \multicolumn{4}{|l|}{ T-score of basal BMD value } \\
\hline L1 & $-0.58 \pm 0.95$ & $-0.75 \pm 0.95$ & 0.112 \\
\hline L2 & $-0.45 \pm 0.94$ & $-0.61 \pm 1.00$ & 0.112 \\
\hline L3 & $-0.40 \pm 0.98$ & $-0.49 \pm 0.92$ & 0.358 \\
\hline L4 & $-0.16 \pm 0.98$ & $-0.68 \pm 1.18$ & $<0.001$ \\
\hline FN & $-0.24 \pm 0.85$ & $-0.40 \pm 0.97$ & 0.105 \\
\hline FT & $-0.23 \pm 1.04$ & $-0.35 \pm 1.01$ & 0.312 \\
\hline \multicolumn{4}{|c|}{ After 1 year } \\
\hline \multicolumn{4}{|l|}{ Biochemical markers } \\
\hline $\mathrm{Ca}(\mathrm{mg} / \mathrm{dL})$ & $9.454 \pm 0.206$ & $9.442 \pm 0.185$ & 0.587 \\
\hline$P(\mathrm{mg} / \mathrm{dL})$ & $3.638 \pm 0.275$ & $3.629 \pm 0.171$ & 0.705 \\
\hline OSC (ng/ml) & $17.797 \pm 2.291$ & $18.424 \pm 2.215$ & 0.012 \\
\hline ALP (IU/L) & $123.609 \pm 4.728$ & $122.777 \pm 5.083$ & 0.121 \\
\hline DPL (pmol/umol creatinine) & $8.408 \pm 0.657$ & $8.726 \pm 0.762$ & $<0.001$ \\
\hline \multicolumn{4}{|l|}{ T-score of BMD value } \\
\hline L1 & $-0.68 \pm 0.82$ & $-0.97 \pm 0.96$ & 0.003 \\
\hline L2 & $-0.33 \pm 1.06$ & $-0.84 \pm 0.83$ & $<0.001$ \\
\hline L3 & $-0.41 \pm 1.07$ & $-0.68 \pm 0.94$ & 0.013 \\
\hline L4 & $-0.18 \pm 0.97$ & $-0.75 \pm 1.15$ & $<0.001$ \\
\hline FN & $-0.28 \pm 0.94$ & $-0.66 \pm 0.91$ & $<0.001$ \\
\hline FT & $-0.38 \pm 0.88$ & $-0.48 \pm 0.77$ & 0.266 \\
\hline
\end{tabular}

Table 3. Comparison of the frequencies of osteoporosis, osteopenia, and normal BMD according to bone mass of the lumbar spine, femur neck, and femur trochanter at baseline and after one year between patients with cervical cancer and controls.

\begin{tabular}{llllll}
\hline & & Control $(\mathrm{N}=225)$ & Cervical cancer $(\mathrm{N}=130)$ & $P$ value & Odds Ratio (95\% CI) \\
\hline L1 & $\mathrm{N}(\%)$ & $\mathrm{N}(\%)$ & & \\
& Osteoporosis & $6(2.7)$ & $6(4.6)$ & $0.368^{*}$ & $1.77(0.56-5.59)$ \\
& Osteopenia & $66(29.3)$ & $45(34.6)$ & 0.301 & $1.27(0.80-2.02)$ \\
L2 & Normal & $153(68.0)$ & $79(60.8)$ & 0.168 & $0.73(0.47-1.14)$ \\
& Osteoporosis & $10(4.4)$ & $4(3.1)$ & 0.524 & $0.68(0.21-2.22)$ \\
& Osteopenia & $48(21.3)$ & $42(32.3)$ & $\mathbf{0 . 0 2 2}$ & $\mathbf{1 . 7 6 ( 1 . 0 8 - 2 . 8 6 )}$ \\
& Normal & $167(74.2)$ & $84(64.6)$ & 0.055 & $0.63(0.39-1.02)$ \\
& Osteoporosis & $9(4.0)$ & $3(2.3)$ & $0.547^{*}$ & $0.57(0.15-2.13)$ \\
& Osteopenia & $46(20.4)$ & $34(26.2)$ & 0.215 & $1.38(0.83-2.29)$ \\
& Normal & $170(75.6)$ & $93(71.5)$ & 0.405 & $0.81(0.50-1.32)$ \\
\hline
\end{tabular}




\begin{tabular}{|c|c|c|c|c|c|}
\hline & & Control $(\mathrm{N}=225)$ & Cervical cancer $(\mathrm{N}=130)$ & $P$ value & Odds Ratio (95\% CI) \\
\hline & & $\mathrm{N}(\%)$ & $\mathrm{N}(\%)$ & & \\
\hline \multirow[t]{3}{*}{$\mathrm{L} 4$} & Osteoporosis & $1(0.4)$ & $13(10.0)$ & $<0.001$ & $24.89(3.22-192.60)$ \\
\hline & Osteopenia & $54(24.0)$ & $36(27.7)$ & 0.441 & $1.21(0.74-1.98)$ \\
\hline & Normal & $170(75.6)$ & $81(62.3)$ & 0.008 & $0.53(0.33-0.85)$ \\
\hline \multirow[t]{3}{*}{$\mathrm{FN}$} & Osteoporosis & $5(2.2)$ & $5(3.8)$ & $0.507^{*}$ & $1.76(0.50-6.20)$ \\
\hline & Osteopenia & $47(20.9)$ & $30(23.1)$ & 0.630 & $1.14(0.68-1.91)$ \\
\hline & Normal & $173(76.9)$ & $95(73.1)$ & 0.421 & $0.82(0.50-1.34)$ \\
\hline \multirow[t]{4}{*}{ FT } & Osteoporosis & $4(1.8)$ & $2(1.5)$ & $1.000^{*}$ & $0.86(0.16-4.78)$ \\
\hline & Osteopenia & $69(30.7)$ & $35(26.9)$ & 0.455 & $0.83(0.51-1.35)$ \\
\hline & Normal & $152(67.6)$ & 93 (71.5) & 0.434 & $1.21(0.75-1.94)$ \\
\hline & \multicolumn{5}{|c|}{ After 1 year } \\
\hline \multirow[t]{3}{*}{ L1 } & Osteoporosis & $7(3.1)$ & $6(4.6)$ & $0.560^{*}$ & $1.51(0.49-4.58)$ \\
\hline & Osteopenia & $62(27.6)$ & $68(52.3)$ & $<0.001$ & $2.88(1.83-4.53)$ \\
\hline & Normal & $156(69.3)$ & $56(43.1)$ & $<0.001$ & $0.34(0.21-0.52)$ \\
\hline \multirow[t]{3}{*}{ L2 } & Osteoporosis & $13(5.8)$ & $5(3.8)$ & 0.424 & $0.65(0.23-1.87)$ \\
\hline & Osteopenia & $52(23.1)$ & $42(32.3)$ & 0.058 & $1.59(0.98-2.57)$ \\
\hline & Normal & $160(71.1)$ & $83(63.8)$ & 0.156 & $0.72(0.45-1.14)$ \\
\hline \multirow[t]{3}{*}{ L3 } & Osteoporosis & $20(8.9)$ & $6(4.6)$ & 0.137 & $0.50(0.19-1.27)$ \\
\hline & Osteopenia & $51(22.7)$ & $48(36.9)$ & 0.004 & $2.00(1.24-3.21)$ \\
\hline & Normal & $154(68.4)$ & $76(58.5)$ & 0.058 & $0.65(0.42-1.02)$ \\
\hline \multirow[t]{3}{*}{ L4 } & Osteoporosis & $7(3.1)$ & $3(2.3)$ & $0.752^{*}$ & $0.74(0.19-2.90)$ \\
\hline & Osteopenia & $45(20.0)$ & $56(43.1)$ & $<0.001$ & $3.03(1.88-4.88)$ \\
\hline & Normal & 173 (76.9) & $71(54.6)$ & $<0.001$ & $0.36(0.23-0.58)$ \\
\hline \multirow[t]{3}{*}{ FN } & Osteoporosis & $4(1.8)$ & $4(3.1)$ & $0.471^{*}$ & $1.75(0.43-7.14)$ \\
\hline & Osteopenia & $58(25.8)$ & 47 (36.2) & 0.039 & $1.63(1.02-2.60)$ \\
\hline & Normal & $163(72.4)$ & 79 (60.8) & 0.023 & $0.59(0.37-0.93)$ \\
\hline \multirow[t]{3}{*}{$\mathrm{FT}$} & Osteoporosis & $4(1.8)$ & $0(0.0)$ & $0.301^{*}$ & . \\
\hline & Osteopenia & $66(29.3)$ & $38(29.2)$ & 0.984 & $0.99(0.62-1.60)$ \\
\hline & Normal & 155 (68.9) & $92(70.8)$ & 0.711 & $1.09(0.68-1.75)$ \\
\hline
\end{tabular}

$P$ values by chi-square test and Fisher's exact test*.

FN, femur neck; FT, femur trochanter.

Table 4. Comparisons of serum and urine biochemical markers, and T-scores of bone mineral density in the lumbar spine, femur neck, and femur trochanter at baseline and after one year between patients with endometrial cancer and controls.

\begin{tabular}{|c|c|c|c|}
\hline & Control & Endometrial Cancer & $P$ value \\
\hline & $\mathrm{N}=225$ & $\mathrm{~N}=68$ & \\
\hline \multicolumn{4}{|c|}{ At baseline } \\
\hline \multicolumn{4}{|l|}{ Biochemical markers } \\
\hline $\mathrm{Ca}(\mathrm{mg} / \mathrm{dL})$ & $9.446 \pm 0.219$ & $9.497 \pm 0.207$ & 0.088 \\
\hline$P(\mathrm{mg} / \mathrm{dL})$ & $3.745 \pm 0.237$ & $3.721 \pm 0.181$ & 0.370 \\
\hline OSC (ng/ml) & $17.342 \pm 2.303$ & $16.895 \pm 1.717$ & 0.086 \\
\hline $\operatorname{ALP}(I U / L)$ & $122.524 \pm 4.713$ & $122.750 \pm 3.911$ & 0.720 \\
\hline DPL (pmol/umol creatinine) & $8.340 \pm 0.817$ & $8.552 \pm 0.735$ & 0.056 \\
\hline \multicolumn{4}{|l|}{ T-score of basal BMD value } \\
\hline L1 & $-0.58 \pm 0.95$ & $-0.56 \pm 0.89$ & 0.884 \\
\hline L2 & $-0.45 \pm 0.94$ & $-0.45 \pm 0.88$ & 0.980 \\
\hline L3 & $-0.40 \pm 0.98$ & $-0.40 \pm 0.94$ & 0.985 \\
\hline L4 & $-0.16 \pm 0.98$ & $-0.13 \pm 1.01$ & 0.863 \\
\hline FN & $-0.24 \pm 0.85$ & $-0.12 \pm 0.89$ & 0.322 \\
\hline FT & $-0.23 \pm 1.04$ & $-0.24 \pm 1.00$ & 0.947 \\
\hline \multicolumn{4}{|c|}{ After 1 year } \\
\hline \multicolumn{4}{|l|}{ Biochemical markers } \\
\hline $\mathrm{Ca}(\mathrm{mg} / \mathrm{dL})$ & $9.454 \pm 0.206$ & $9.443 \pm 0.185$ & 0.678 \\
\hline$P(\mathrm{mg} / \mathrm{dL})$ & $3.638 \pm 0.275$ & $3.628 \pm 0.171$ & 0.711 \\
\hline OSC (ng/ml) & $17.797 \pm 2.291$ & $18.236 \pm 2.213$ & 0.164 \\
\hline $\operatorname{ALP}(\mathrm{IU} / \mathrm{L})$ & $123.609 \pm 4.728$ & $121.294 \pm 5.384$ & 0.001 \\
\hline DPL (pmol/umol creatinine) & $8.408 \pm 0.657$ & $8.453 \pm 0.863$ & 0.693 \\
\hline \multicolumn{4}{|l|}{ T-score of BMD value } \\
\hline L1 & $-0.68 \pm 0.82$ & $-0.88 \pm 0.95$ & 0.116 \\
\hline L2 & $-0.33 \pm 1.06$ & $-0.56 \pm 0.88$ & 0.080 \\
\hline L3 & $-0.41 \pm 1.07$ & $-0.89 \pm 0.94$ & $<0.001$ \\
\hline L4 & $-0.18 \pm 0.97$ & $-0.83 \pm 1.16$ & $<0.001$ \\
\hline FN & $-0.28 \pm 0.94$ & $-0.93 \pm 0.81$ & $<0.001$ \\
\hline FT & $-0.38 \pm 0.88$ & $-0.80 \pm 0.90$ & $<0.001$ \\
\hline
\end{tabular}

Values are mean \pm standard deviation. $P$ values by unpaired t-test.

ALP, total alkaline phophatase; BMD, bone mineral density; Ca, calcium; DPL, deoxypiridinoline; FN, femur neck; FT, femur trochanter; OSC, osteocalcin; P, phosphorus. 
Table 5. Comparison of the frequencies of osteoporosis, osteopenia, and normal BMD according to bone mass of the lumbar spine, femur neck, and femur trochanter at baseline and after one year between patients with endometrial cancer and controls.

\begin{tabular}{|c|c|c|c|c|c|}
\hline & & Control $(\mathrm{N}=225)$ & Endometrial cancer $(\mathrm{N}=68)$ & $P$ value & Odds Ratio (95\% CI) \\
\hline & & $\mathrm{N}(\%)$ & $\mathrm{N}(\%)$ & & \\
\hline \multirow[t]{3}{*}{ L1 } & Osteoporosis & $6(2.7)$ & $2(2.9)$ & $1.000^{*}$ & $1.11(0.22-5.61)$ \\
\hline & Osteopenia & $66(29.3)$ & $19(27.9)$ & 0.825 & $0.93(0.51-1.71)$ \\
\hline & Normal & $153(68.0)$ & $47(69.1)$ & 1.000 & $1.05(0.59-1.89)$ \\
\hline \multirow[t]{3}{*}{ L2 } & Osteoporosis & $10(4.4)$ & $2(2.9)$ & $0.739^{*}$ & $0.65(0.14-3.05)$ \\
\hline & Osteopenia & $48(21.3)$ & $15(22.1)$ & 0.898 & $1.04(0.54-2.01)$ \\
\hline & Normal & $167(74.2)$ & $51(75.0)$ & 0.898 & $1.04(0.56-1.95)$ \\
\hline \multirow[t]{3}{*}{ L3 } & Osteoporosis & $9(4.0)$ & $2(2.9)$ & $1.000^{*}$ & $0.73(0.15-3.45)$ \\
\hline & Osteopenia & $46(20.4)$ & $15(22.1)$ & 0.774 & $1.10(0.57-2.13)$ \\
\hline & Normal & $170(75.6)$ & $51(75.0)$ & 0.926 & $0.97(0.52-1.82)$ \\
\hline \multirow[t]{3}{*}{ L4 } & Osteoporosis & $1(0.4)$ & $0(0.0)$ & $1.000^{*}$ & . \\
\hline & Osteopenia & $54(24.0)$ & $17(25.0)$ & 0.866 & $1.06(0.56-1.98)$ \\
\hline & Normal & $170(75.6)$ & $51(75.0)$ & 0.926 & $0.97(0.52-1.82)$ \\
\hline \multirow[t]{3}{*}{ FN } & Osteoporosis & $5(2.2)$ & $2(2.9)$ & $0.665^{*}$ & $1.33(0.25-7.03)$ \\
\hline & Osteopenia & $47(20.9)$ & $10(14.7)$ & 0.259 & $0.65(0.31-1.37)$ \\
\hline & Normal & $173(76.9)$ & $56(82.4)$ & 0.339 & $1.40(0.70-2.81)$ \\
\hline \multirow[t]{3}{*}{ FT } & Osteoporosis & $4(1.8)$ & $1(1.5)$ & $1.000^{*}$ & $0.82(0.09-7.50)$ \\
\hline & Osteopenia & 69 (30.7) & $15(22.1)$ & 0.169 & $0.64(0.34-1.21)$ \\
\hline & Normal & $152(67.6)$ & $52(76.5)$ & 0.161 & $1.56(0.84-2.92)$ \\
\hline \multicolumn{6}{|c|}{ After 1 year } \\
\hline \multirow[t]{3}{*}{ L1 } & Osteoporosis & $7(3.1)$ & $3(4.4)$ & $0.703^{*}$ & $1.44(0.36-5.72)$ \\
\hline & Osteopenia & $62(27.6)$ & $34(50.0)$ & 0.001 & $2.63(1.51-4.59)$ \\
\hline & Normal & $156(69.3)$ & $31(45.6)$ & $<0.001$ & $0.37(0.21-0.65)$ \\
\hline \multirow[t]{3}{*}{ L2 } & Osteoporosis & $13(5.8)$ & $2(2.9)$ & $0.533^{*}$ & $0.49(0.11-2.25)$ \\
\hline & Osteopenia & $52(23.1)$ & $16(23.5)$ & 0.943 & $1.02(0.54-1.94)$ \\
\hline & Normal & $160(71.1)$ & $50(73.5)$ & 0.698 & $1.24(0.61-2.08)$ \\
\hline \multirow[t]{3}{*}{ L3 } & Osteoporosis & $20(8.9)$ & $4(5.9)$ & 0.428 & $0.64(0.21-1.94)$ \\
\hline & Osteopenia & $51(22.7)$ & $32(47.1)$ & $<0.001$ & $3.03(1.72-5.36)$ \\
\hline & Normal & $154(68.4)$ & 32 (47.1) & 0.001 & $0.41(0.24-0.71)$ \\
\hline \multirow[t]{3}{*}{ L4 } & Osteoporosis & $7(3.1)$ & $3(4.4)$ & $0.703^{*}$ & $1.44(0.36-5.72)$ \\
\hline & Osteopenia & $45(20.0)$ & $30(44.1)$ & $<0.001$ & $3.16(1.77-5.64)$ \\
\hline & Normal & $173(76.9)$ & 35 (51.5) & $<0.001$ & $0.32(0.18-0.56)$ \\
\hline \multirow[t]{3}{*}{ FN } & Osteoporosis & $4(1.8)$ & $2(2.9)$ & $0.626^{*}$ & $1.67(0.30-9.35)$ \\
\hline & Osteopenia & $58(25.8)$ & $33(48.5)$ & $<0.001$ & $2.72(1.55-4.76)$ \\
\hline & Normal & $163(72.4)$ & $33(48.5)$ & $<0.001$ & $0.36(0.21-0.63)$ \\
\hline \multirow[t]{3}{*}{ FT } & Osteoporosis & $4(1.8)$ & $2(2.9)$ & $0.626^{*}$ & $1.67(0.30-9.35)$ \\
\hline & Osteopenia & $66(29.3)$ & $31(45.6)$ & 0.013 & $2.02(1.16-3.52)$ \\
\hline & Normal & $155(68.9)$ & 35 (51.5) & 0.008 & $0.48(0.28-0.83)$ \\
\hline
\end{tabular}

$P$ values by chi-square test and Fisher's exact test*.

FN, femur neck; FT, femur trochanter.

Anti-cancer treatments for gynecologic cancers are mainly composed of castrating surgery, radiation, and chemotherapy, all of which could cause hypogonadism and induce CTIBL $[7,17]$. Other mechanisms of CTIBL include direct or indirect effects of cancer therapies or malignancy on bone metabolism, inactivity, and inadequate intake of calcium and vitamin D [7]. Several chemotherapy agents may have direct effects on bone metabolism independent of their effects on gonadal hormones, and high-dose chemotherapy regimens, such as regimens used with hematopoietic stem-cell transplantation (HSCT), are toxic to osteoprogenitor cells in a dose dependent manner. Radiation therapy can lead to atrophic changes of bone which may be complicated by fracture [23]. In addition, other drugs commonly used in cancer patients, such as glucocorticoids, cyclosporins, and L-thyroxine, are also associated with bone loss [7, 17]. Main anti-cancer treatment in the present study comprises (radical) hysterectomy with BSO and RT (CCRT for cervical cancer, and radiotherapy for endometrial cancer). We suggest that RT not surgery plays a decisive role in bone loss developed in patients with gynecologic cancer because the impact of surgery, especially BSO, on endogenous estrogen may be insignificant, for all of the participants in this study are postmenopausal women at baseline.

High dose local radiation therapy was known to be associated with atrophy of the trabeculae of bone which may be complicated by fracture, and in addition, it has an indirect effect on bone associated with vascular changes [23]. Radiation field includes the bony structures of the pelvis in the in women treated for cervical and endometrial cancer, and pelvic irradiation can contribute to the development of pelvic insufficiency fracture [24]. Previous studies have noted that CCRT reduces BMD on women with cervical cancer [18, 19]. Nishio et al. [18] reported that 
patients with cervical cancer who were treated with CCRT after surgical treatment showed a significant decrease in the lumbar spine BMD to $91.9 \pm 5.9 \%$, and Hwang et al. [19] reported that BMDs at L4 and greater trochanter of the femur in women with cervical cancer treated with CCRT were significantly lower than those of the controls. On the contrary, adjuvant chemotherapy in postmenopausal women with endometrial cancer may not be related to CTIBL. Nine patients with endometrial cancer who received a total of three courses of paclitaxel plus carboplatin chemotherapy following surgery showed no significant decrease of spinal BMD compared to basal value [18].

In the present study, T-scores of basal BMD only in L4 were significantly lower in patients with cervical cancer compared to those in controls. One possibility is that L4 is the most vulnerable segment of the lumbar spine to bone loss related to cervical cancer, which is partially in agreement with the result of Hwang et al [19]. The association between cervical cancer and decreased basal BMD of the lumbar spine before anti-cancer treatment has been noted in few studies. Two studies reported that patients with cervical cancer without bone metastases had significantly lower BMD in the LS compared to controls [13, 14]. On the contrary, Lee et al. [25] reported that total femoral $\mathrm{BMD}$, not spinal BMD, was significantly lower in patients with cervical cancer compared to controls, and it is contrary to other studies including ours.

Cancer-induced bone loss (CIBL) may develop by osteolytic factors secreted by human cancer cell lines [6]. Several factors are known to be related to the activation of osteoclasts by tumor cells including parathyroid hormone-like peptide [8-10] transforming growth factor [11], osteoclast activating factor [12], and prostaglandins [13]. In cancer patients, greater osteoclastic activity, markedly reduced osteoblastic surface, osteoid surface, and osteoid volume have been noted by quantitative histochemical studies of the bone $[6,26]$. If the reduced bone mass in patients with cancer was related to the bone-resorbing factors secreted by cancer-cell lines, we would expect to develop hypercalcemia. In our study, serum calcium levels were borderline significantly higher in patients with cervical cancer compared to controls $(P=0.049)$, but this hypercalcemic condition in cervical cancer group was not observed after cancer treatment. Cho et al. [13] suggested one possible explanation that some cases of malignancy may have been associated with elevated levels of bone-resorbing materials even in the absence of hypercalcemia because of regulatory mechanisms that maintain normocalcemia, as proposed by Henderson et al. [27].

In the present study, there were no significant differences of basal BMD in the LS and femur between patients with endometrial cancer and controls, and it is similar to the result of Lee et al. [15]. We hypothesized that BMD values at the LS and femur were not different between two groups because bone mass in patients with endometrial cancer may have reached a balance between the negative effect of CIBL and the positive effect of high endogenous estrogen levels related to endometrial cancer [28]. The persistent influence of estrogen can increase basal bone mass and reduce fracture risk.

Biochemical bone turnover markers are indicators of bone metabolism, both the formation and resorption of bone [29]. In the present study, serum OSC and urine DPL were lower in patients with cervical cancer than in controls after cancer treatment, and total ALP levels were lower in endometrial cancer group after cancer treatment than in controls. Lower level of total ALP in in endometrial cancer group is the opposite to Hwang et al. [19] who reported significant higher levels of total ALP in patients with cervical cancer who received anti-cancer treatment compared to controls. This discrepancy between two studies remains hard to explain.

Although our study had a relatively larger sample size compared to that in other studies that examined BMD in patients with gynecologic cancer after cancer treatment $[18,19]$, limitations of this study mainly stem from its retrospective observational study design. All patients with gynecologic cancer in the present study did not receive the same anti-cancer treatment. In addition, we did not consider other confounding factors related to BMD which may provide a clearer association between anti-cancer treatment for gynecologic cancer and bone density.

\section{Acknowledgements}

This research was supported by Basic Science Research Program through the National Research Foundation of Korea (NRF) funded by the Ministry of Education (2013R1A1A4A01 010141).

\section{Competing Interests}

The authors have declared that no competing interest exists.

\section{References}

1. [No authors listed]. Osteoporosis prevention, diagnosis, and therapy. NIH Consensus Statement. 2000; 17: 1-45.

2. Fritz MA, Speroff L. Clinical gynecologic endocrinology and infertility, 8th ed. Philadelphia, USA: Lippincott Williams \& Wilkins; 2011: 713-7.

3. Orwoll ES, Klein RF. Osteoporosis in men. Endocr Rev. 1995; 16: 87-116.

4. Lane NE. Epidemiology, etiology, and diagnosis of osteoporosis. Am J Obstet Gynecol. 2006; 194: S3-S11.

5. Shifren JL, Schiff I. Menopause. In: Berek JS, ed. Berek \& Novak's Gynecology, 15th ed. Philadelphia: Lippincott Williams \& Wilkins; 2012: 1239-42.

6. Mundy GR, Eilon G, Orr W, Spiro TP, Yoneda T. Osteoclast activating factor: its role in myeloma and other types of hypercalcemia of malignancy. Metab Bone Dis Relat Res. 1980; 2: 173-7.

7. Michaud LB, Goodin S. Cancer-treatment induced bone loss, part 1. Am J Health Syst Pharm. 2006; 63: 419-430. 
8. Suva LJ, Winslow GA, Wettenhall RE, et al. A parathyroid hormone-related protein implicated in malignant hypercalcemia: Cloning and expression. Science. 1987; 237: 893-6.

9. Stewart AF, Wu T, Goumas D, Burtis WJ, Broadus AE. N-terminal amino acid sequence of two novel tumor-derived adenylate cyclase-stimulating proteins: Identification of parathyroid hormone-like and parathyroid hormone-unlike domains. Biochem Biophys Res Commun. 1987; 146: 672-8.

10. Strewler GJ, Stern PH, Jacobs JW, et al. Parathyroid hormone like protein from human renal carcinoma cells. Structural and functional homology with parathyroid hormone. J Clin Invest. 1987; 80: 1803-7.

11. Sherwin SA, Twardzik DR, Bohn WH, Cockley KD, Todaro GJ High-molecular-weight transforming growth factor activity in the urine of patients with disseminated cancer. Cancer Res. 1983; 43: 403-7.

12. Seyberth HW, Segre GV, Morgan JL, Sweetman BJ, Potts JT Jr, Oates JA. Prostaglandins as mediators of hypercalcemia associated with certain types of cancer. N Engl J Med. 1975; 293: 1278-83.

13. Cho SH, Cho SH, Lee JA, Moon H, Kim DS. Reduced spinal bone mass in patients with uterine cervical cancer. Obstet Gynecol. 1991; 78: 689-92.

14. Hung YC, Yeh LS, Chang WC, Lin CC, Kao CH. Prospective study of decreased bone mineral density in patients with cervical cancer without bone metastases: A preliminary report. Jpn J Clin Oncol. 2002; 32: 422-4

15. Lee SH, Ku CH, Shin JW, Park JM, Park CY. Bone Mineral Density in Patients with Endometrial Cancer. J Korean Soc Menopause. 2009; 15: 35-40.

16. McGlynn KA, Gridley G, Mellemkjaer L, et al. Risks of cancer among a cohort of 23,935 men and women with osteoporosis. Int J Cancer. 2008; 122: 1879-84.

17. Pfeilschifter J, Diel IJ. Osteoporosis Due to Cancer Treatment: Pathogenesis and Management. J Clin Oncol. 2000; 18: 1570-1593.

18. Nishio K, Tanabe A, Maruoka R, et al. Bone mineral loss induced by anticancer treatment for gynecological malignancies in premenopausal women. Endocr Connect. 2012; 2: 11-7.

19. Hwang JH, Song $\mathrm{SH}$, Lee JK, Lee NW, Lee KW. Bone mineral density after concurrent chemoradiation in patients with uterine cervical cancer. Menopause. 2010; 17: 416-20

20. Kanis JA, Melton LJ 3rd, Christiansen C, Johnston CC, Khaltaev N. The diagnosis of osteoporosis. J Bone Miner Res. 1994; 9: 1137-41.

21. Adler RA. Cancer treatment-induced bone loss. Curr Opin Endocrinol Diabetes Obes. 2007; 14: 442-5.

22. Brufsky AM. Cancer treatment-induced bone loss: pathophysiology and clinical perspectives. Oncologist. 2008; 13: 187-95.

23. Howland WJ, Loeffler RK, Starchman DE, Johnson RG. Postirradiation atrophic changes of bone and related complications. Radiology. 1975; 117:677-85.

24. Oh D, Huh SJ, Nam H, et al. Pelvic insufficiency fracture after pelvic radiotherapy for cervical cancer: analysis of risk factors. Int J Radiat Oncol Biol Phys. 2008; 70: 1183-8.

25. Lee SH, Ku CH, Lee KB, Shin JW, Park CY. Decreased bone mineral density of femur in patients with cervical cancer. J Obstet Gynaecol Res. 2009; 35: 335-8.

26. Stewart AF, Vignery A, Silverglate A, et al Quantitative bone histomorphometry in humoral hypercalcemia of malignancy: Uncoupling of bone cell activity. J Clin Endocrinol Metab. 1982; 55: 219-27.

27. Henderson JE, Shustik C, Kremer R, Rabbani SA, Hendy GN, Goltzman D. Circulating concentrations of parathyroid hormone-like peptide in malignancy and in hyperparathyroidism. J Bone Miner Res. 1990; 5: 105-13.

28. Dowdy SC, Mariani A, Lurain JR. Uterine Cancer. In: Berek JS, ed. Berek \& Novak's Gynecology, 15th ed. Philadelphia: Lippincott Williams \& Wilkins; 2012: 1250-2.

29. Demers LM, Costa L, Chinchilli VM, Gaydos L, Curley E, Lipton A. Biochemical markers of bone turnover in patients with metastatic bone disease. Clin Chem. 1995; 41: 1489-94. 